\title{
PERFORMANCE COMPARISON OF BLIND ADAPTIVE MULTIUSER DETECTION ALGORITHMS
}

\author{
Khalifa Hunki ${ }^{1}$, EhabM. Shaheen ${ }^{2}$, Mohamed Soliman ${ }^{3}$, K. A. El-Barbary ${ }^{4}$ \\ ${ }^{1,2,3}$ Military Technical College, Cairo, Egypt \\ ${ }^{4}$ Suez Canal University, Suez, Egypt \\ hunki07@gmai.com,efareed2007@yahoo.com,mnna3000@yahoo.com,khbar2000@yahoo.com
}

\begin{abstract}
Blind multiuser detection algorithms are used to eliminate the Multiple Access Interference (MAI) and the Near-Far effect in mobile communication systems. Four kinds of blind multiuser detection algorithms applied to code-division multiple-access (CDMA) communication system are studied in this paper. Those algorithms are the Least Mean Squares (LMS), Recursive Least Squares $(R L S)$, Kalman filter and subspace-based Kalman filter algorithms. The resultant signal to interference ratio (SIR) at the output of the receivers controlled by the four kinds of multi-user detection algorithm has been discussed in this paper. Simulation results show that the subspace based Kalman filter algorithm outperforms all other three algorithms. Subspace-based Kalman filter algorithm has faster convergence speed, more practical and the capability of CDMA system can be increased.
\end{abstract}

Keywords: Blind multiuser detection, Code-division multiple access, Mean output energy, Adaptive filtering.

\section{INTRODUCTION}

Direct-sequence code-division multiple-access (DS-CDMA) has been widely studied in the literatures. Recently, adaptive interference suppression techniques based on multiuser detection have been considered as powerful methods for increasing the quality, capacity, and coverage of CDMA systems [1].

The mitigation of MAI in CDMA systems is a problem of continuing interest since MAI is the dominant impairment for CDMA systems. It is widely recognized that MAI exists even in perfect power-controlled CDMA systems [2]. Multiuser detectors perform better than the conventional detector under all power distributions, except in pathological cases, such as the decorrelating detector in extremely low signal to noise ratio (SNR) [2]. Therefore, multiuser detection is not only a solution to the near-far problem but is also useful even with power control.

In order to successfully eliminate the MAI and detect the desired user's information bits, one or more of the following is usually required at the receiver end:

1) Spreading waveform of the desired user;

2) Spreading waveforms of the interfering users;

3) Propagation delay (timing) of the desired user;

4) Propagation delays of the interfering users;

5) Received amplitudes of the interfering users (relative to that of the desired user);

6) Training data sequences for every active user
The "blind" adaptive multiuser detectors require only the knowledge of (1) and (3), which is, the same knowledge as the conventional receiver.

Previous work on blind adaptive multiuser detection dates back to a 1995 paper by Honig et al. [3], who established a canonical representation for blind multiuser detectors and used stochastic gradient algorithms such as LMS to implement the blind adaptive mean output energy (MOE) detector. As elegantly shown by Roy [4], the blind adaptive MOE detector has a smaller eigenvalue spread than the training-based adaptive LMS detector; hence, the blind LMS algorithm always provides (nominally) faster convergence than the training driven LMS-MMSE receiver but at the cost of increased tap-weight fluctuation or misadjustment.

It is well-known that the RLS algorithm and the Kalman filtering algorithm are better than the LMS algorithm in convergence rate and tracking capability [2]. Using the exponentially weighted sum of error squares cost function, Chen and Roy [5] proposed an RLS algorithm that requires the knowledge of (1)-(4) and, thus, is not a blind multiuser detector. Later, Poor and Wang [6] proposed an exponentially windowed RLS algorithm for blind multiuser detection requiring only the knowledge of (1) and (3).

On the other hand, the RLS is a special case of the Kalman filter [7], [8], whereas the Kalman filter is known to be a linear minimum variance state estimator [7], [9] and [10]. Motivated probably by these two facts, some attention has been focused on Kalman filter-based adaptive multiuser 
detection [11]-[15]. In particular, it is shown in [13] that when applied in an asynchronous CDMA system, the RLS algorithm performs more poorly than the more general Kalman filter algorithm.

Zhang and Wei [2] proposed a simple and effective state space model for the multiuser detection problem in a stationary or slowly fading channel and employed Kalman filter as the adaptive algorithm. Compared with the LMS approach in [3] and the RLS approach in [6], this detector demonstrates lower steady-state excess output energy in adaptation. Interestingly, though the state space model for the Kalman filter was devised under a time-invariant assumption, the resulting algorithm could work well in a slowly time-varying environment.

Motivated by the signal subspace concept in [16], Zhou et al. [17] proposed a modified version of this blind adaptive multiuser detector by modeling the detector as a vector in the signal subspace and employing a Kalman filter philosophy similar to that in [2] to derive the coefficients adaptively.

Compared with the full-rank approach in [2], despite some similarity in the state-space model, this new subspace-based multiuser detector has some significantly important merits. First it has lower computational complexity and faster convergence rate in terms of SIR. Secondly, it is less conditioned on some system parameters such as the desired users' signal amplitude than the full-rank method, thus it is a blind detection method in a more strict sense. Additionally, the detection effectiveness is maintained both in additive Gaussian noise channels and in slowly time-varying Rayleigh fading channels. In a dynamical system where users can enter and leave at random, the structure of the signal subspace is also time-varying. In this case a subspace tracking algorithm is seamlessly integrated into the proposed detector to track the changes and provide an online estimation of the signal subspace.

To this end; this paperpresents and comparesthe four mentioned algorithms. Their performances in the case of static and dynamic channels are presented and comments are provided to fulfill the comparative study.

\section{SIGNAL MODEL}

Consider an antipodal K-user synchronous DS-CDMA system signaling through an additive white Gaussian noise channel. By passing through a chip-matched filter, followed by a chiprate sampler, the discrete-time output of the receiver during one symbol interval can be modeled as

$$
\begin{array}{r}
r(n)=\sum_{k=1}^{K} A_{k} b_{k}(n) s_{k}(n)+\sigma v(n), \\
n=0,1, \ldots \ldots T_{s}-1
\end{array}
$$

Where

$v(n)$ ambient channel noise;

$K$ number of users;

$A_{k}$ received amplitude of the $k^{\text {th }}$ user;

$b_{k}(n)$ information symbol sequence from the $k^{\text {th }}$ user, chosen independentlyand equally from $\{-1,+1\}$;

$s_{k}(n)$ signature waveform of the $k^{\text {th }}$ user;

It is assumed that $s_{k}(n)$ is supported only on the interval $\left[0, T_{s}-1\right]$,

Where

$T_{s}=N T_{c}$ symbol interval;

$T_{c}$ chip interval;

$N$ processing gain.

Defining

$\boldsymbol{r}(n)=[r(0), r(1), \ldots, r(N-1)]^{T}$
$\boldsymbol{v}(n)=[v(0), v(1), \ldots, v(N-1)]^{T}$

We can express (1) in vector form

$\boldsymbol{r}(n)=A_{1} b_{1}(n) \boldsymbol{s}_{1}+\sum_{k=2}^{K} A_{k} b_{k}(n) \boldsymbol{s}_{k}+\sigma \boldsymbol{v}(n)(3)$

Where

$\boldsymbol{s}_{k}=(1 / \sqrt{N})\left[s_{k}(0), s_{k}(1), \ldots, s_{k}(N-1)\right]^{T}$ is the code sequence assigned to the $k^{\text {th }}$ user.

For convenience, we will assume that the desired user is $k=1$. It is well known that any linear multiuser detector for user 1 can be characterized by the tap-weight vector $\boldsymbol{c}_{1}(n)$ such that the decision on $b_{1}(n)$ during the $n^{\text {th }}$ symbol interval is given by

$$
\hat{b}_{1}(n)=\operatorname{sgn}\left(\left\langle\boldsymbol{c}_{1}, \boldsymbol{r}\right\rangle\right)=\operatorname{sgn}\left(\boldsymbol{c}_{1}^{T}, \boldsymbol{r}(n)\right)(4)
$$

Where $\langle\boldsymbol{a}, \boldsymbol{b}\rangle$ denotes the dot product of the vectors $\boldsymbol{a}, \boldsymbol{b}$

\section{BLIND ADAPTIVE MULTIUSER DETECTORS}

\subsection{Blind LMS Multiuser Detector}

The canonical representation of a linear blind adaptive multiuser detector for user 1 was firstly established [3] as follows:

$$
c_{1}(n)=s_{1}+x_{1}(n)(5)
$$

Subject to

$$
\left\langle s_{1}, x_{1}\right\rangle=0
$$


Where $\boldsymbol{s}_{1}$ is the spreading vector of the first user and $\boldsymbol{c}_{1}(n)$ is the adaptive part of the detector. By minimizing a MOE cost function of the form.

$$
\operatorname{MOE}\left(\boldsymbol{c}_{1}\right)=E\left\{\left\langle\boldsymbol{r}, \boldsymbol{c}_{1}\right\rangle^{2}\right\}(7)
$$

Honig et al. [3] have proposed a blind LMS based algorithm to update the adaptive

$$
\boldsymbol{x}_{1}(n)=\boldsymbol{x}_{1}(n-1)-\mu z(n)\left[\boldsymbol{r}(n)-z_{M F}(n) \boldsymbol{s}_{1}\right](8)
$$

Wherez $(n)=\left\langle\boldsymbol{r}(n), \boldsymbol{s}_{1}+\boldsymbol{x}_{1}(n-1)\right\rangle$ is the output of the detector $z_{M F}(n)=\left\langle\boldsymbol{r}(n), \boldsymbol{s}_{1}\right\rangle$ is the output of the conventional matched-filter, and $\mu$ is the step-size that controls the adaptation speed. The step size is given by (70) in [3], when implementing the LMS algorithm, the step-size must satisfy the stability condition of convergence of output MSE:

$$
\mu<\frac{2}{\sum_{k=1}^{K} A_{k}^{2}+N \sigma^{2}}
$$

\subsection{Blind RLS Multiuser Detector}

Using the minimum output energy criterion, Poor and Wang [6] proposed a RLS algorithm for blind multiuser detection. The exponentially windowed RLS algorithm selects the weight vector to minimize the sum of exponentially weighted output energy, namely

$\operatorname{minimize} \sum_{i=1}^{n} \lambda^{n-1}\left(\boldsymbol{c}_{1}^{T}(n) \boldsymbol{r}(n)\right)^{2}$

Subject to

$$
\boldsymbol{s}_{1}^{T} \boldsymbol{c}_{1}(n)=1
$$

Where $0<\lambda>1$ is the forgetting factor. The solution to this constrained optimization problem yields the linear MMSE detector, which is given by [4], [11]

Where

$$
\boldsymbol{c}_{1}(n)=\frac{\boldsymbol{R}^{-1}(n) \boldsymbol{s}_{1}}{\boldsymbol{s}_{1}^{T} \boldsymbol{R}^{-1}(n) \boldsymbol{s}_{1}}
$$

$$
\boldsymbol{R}(n)=\sum_{i=1}^{n} \lambda^{n-i} \boldsymbol{r}(i) \boldsymbol{r}^{T}(i)
$$

A recursive procedure for $\boldsymbol{c}(n)$ updating can be obtained as follows:

$$
\boldsymbol{k}(n) \triangleq \frac{\boldsymbol{R}^{-1}(n-1) \boldsymbol{r}(n)}{\lambda+\boldsymbol{r}^{T}(n) \boldsymbol{R}^{-1}(n) \boldsymbol{r}(n)}
$$

$$
\begin{gathered}
\frac{1}{\lambda}\left[\boldsymbol{h}(n-1)-\boldsymbol{k}(n) \boldsymbol{r}^{T}(n) \boldsymbol{h}(n-1)\right] \\
\boldsymbol{c}(n)=\frac{1}{\boldsymbol{s}^{T} \boldsymbol{h}(n)} \boldsymbol{h}(n) \\
\boldsymbol{R}^{-1}(n)=\frac{1}{\lambda}\left[\boldsymbol{R}^{-1}(n-1)-\boldsymbol{k}(n) \boldsymbol{r}^{T}(n) \boldsymbol{R}^{-1}(n-1)\right]
\end{gathered}
$$

\subsection{Blind Multiuser Detection Based on Kalman Filtering}

In [2], Zhang et al. have proposed to use an alternative standard representation for the blind adaptive multiuser detector:

$$
\boldsymbol{c}_{1}(n)=\boldsymbol{s}_{1}-\boldsymbol{C}_{1, \text { null }} \boldsymbol{w}_{1}(n)
$$

Where the columns of the $N \times N^{-1}$ matrix $\boldsymbol{C}_{1, \text { null }}$ span the null space of $\boldsymbol{s}_{1}$, i.e

$$
\boldsymbol{s}_{1}^{T} \boldsymbol{C}_{1, \text { null }}=0
$$

It should be noted that $\boldsymbol{C}_{1, \text { null }}$ can be pre-computed off-line via one of many orthogonalization procedures such as the GramSchmidt orthogonalization. Unlike (5), the adaptive part $\boldsymbol{w}_{1}(n)$ in $(18)$ is now of size $(N-1) \times 1$ and has the advantage of being unconstraint. Let us define the output of the detector as follows:

$$
z(n)=\boldsymbol{c}_{1}^{T}(n) \boldsymbol{r}(n)
$$

Then $z(n)$ has zero-mean and its variance is given by Paragraph comes content here.

$$
E\left\{z^{2}(n)\right\}=\operatorname{MOE}\left(\boldsymbol{c}_{1}(n)\right)=\operatorname{MSE}\left(\boldsymbol{c}_{1}(n)\right)+A_{1}^{2}
$$

Thus, when the detector is optimal (i.e., $\operatorname{MSE}\left(\boldsymbol{c}_{1}(n)\right)$ attains its MMSE value), the variance of $z(n)$ corresponds to the minimum MOE and is dominated by the power of the desired user $A_{1}^{2}$.

Substituting (18) in (20) yields

$$
z(n)=\boldsymbol{s}_{1}^{T} \boldsymbol{r}(n)-\boldsymbol{r}^{T}(n) \boldsymbol{C}_{1, n u l l} \boldsymbol{w}_{1}(n)
$$

Put $\quad z_{M F}(n)=\boldsymbol{s}_{1}^{T} \boldsymbol{r}(n)$ and $\quad \boldsymbol{d}^{T}(n)=\boldsymbol{r}^{T}(n) \boldsymbol{C}_{1, n u l l} . \quad$ If $\boldsymbol{w}_{1}$ achieves $\boldsymbol{w}_{\text {opt } 1}$, then (22) can be rewritten as the following measurement equation:

$$
z_{M F}(n)=\boldsymbol{d}^{T}(n) \boldsymbol{w}_{o p t 1}(n)+z(n)
$$

$\boldsymbol{h}(n) \triangleq \boldsymbol{R}^{-1}(n) \boldsymbol{s}_{1}=$ 
If the detector is assumed to be time-invariant, one can write:

$$
\boldsymbol{w}_{o p t 1}(n)=\boldsymbol{w}_{o p t 1}(n-1)
$$

As (23) and (24) define a state-space representation of the adaptive part of the detector, Kalman filtering makes it possible to recursively update $\boldsymbol{w}(n)[2]$.

$$
\begin{aligned}
& \boldsymbol{g}(n)=\boldsymbol{K}(n, n-1) \boldsymbol{d}(n) \\
& \times\left[\boldsymbol{d}^{T}(n) \boldsymbol{K}(n, n-1) \boldsymbol{d}(n)+\xi_{m i n}\right]^{-1} \\
& \boldsymbol{K}(n+1, n)=\boldsymbol{K}(n, n-1) \\
& -\boldsymbol{g}(n) \boldsymbol{d}^{T}(n) \boldsymbol{K}(n, n-1) \\
& \widehat{\boldsymbol{w}}_{\text {opt } 1}(n)=\widehat{\boldsymbol{w}}_{\text {opt } 1}(n-1) \\
& +\boldsymbol{g}(n)\left[z_{M F}(n)-\boldsymbol{d}^{T}(n) \widehat{\boldsymbol{w}}_{o p t 1}(n-1)\right] \\
& \boldsymbol{c}_{1}(n)=\boldsymbol{s}_{1}-\boldsymbol{C}_{1, n u l l} \widehat{\boldsymbol{w}}_{\text {opt } 1}(n)
\end{aligned}
$$

Where $\boldsymbol{g}(n)$ is $(N-1) \times 1$ Kalman gain vector, $K(n+1, n)$ is $(N-1) \times(N-1)$ correlation matrix of predicted state error and $\xi_{\text {min }}=\operatorname{MOE}\left(\boldsymbol{c}_{1}(n)\right)$ is the minimum MOE of the dynamical system of user 1 . The initial value $\widehat{\boldsymbol{w}}_{\text {opt } 1}(0)=0$ and $\boldsymbol{K}(1,0)=$ I.

\subsection{Subspace-based Blind Multiuser Detector using}

\section{Kalman Filter}

Based on the signal model (3) and the associated independent assumptions, the autocorrelation matrix of the received signal $\boldsymbol{r}(n)$ can be expressed as

$$
\begin{gathered}
\boldsymbol{R}=E \\
=\boldsymbol{S} \boldsymbol{\boldsymbol { A }}(n) \boldsymbol{r}(n)^{T}+\sigma^{2} \boldsymbol{I}_{N}
\end{gathered}
$$

Where $\boldsymbol{S}=\left[\begin{array}{llll}\boldsymbol{s}_{1} \boldsymbol{s}_{2} & \ldots & \boldsymbol{s}_{K}\end{array}\right]$ denotes the signature matrix, and $\boldsymbol{A}=\operatorname{diag}\left(A_{1}^{2} A_{2}^{2} \ldots A_{K}^{2}\right)$ denotes the diagonal matrix of the signal amplitude. On the other hand, applying an eigende composition to the matrix $\boldsymbol{R}$ yields

$$
\boldsymbol{R}=\boldsymbol{U} \boldsymbol{\Lambda} \boldsymbol{U}^{T}=\boldsymbol{U}_{s} \boldsymbol{\Lambda}_{s} \boldsymbol{U}_{s}^{T}+\boldsymbol{U}_{n} \boldsymbol{\Lambda}_{n} \boldsymbol{U}_{n}^{T}
$$

where $\quad \boldsymbol{U}=\left[\begin{array}{ll}\boldsymbol{U}_{s} & \boldsymbol{U}_{n}\end{array}\right], \quad \boldsymbol{\Lambda}=\operatorname{diag}\left(\boldsymbol{\Lambda}_{s} \quad \boldsymbol{\Lambda}_{n}\right) . \quad \boldsymbol{\Lambda}_{\mathrm{s}}=$ $\operatorname{diag}\left(\lambda_{1} \lambda_{2} \ldots \lambda_{\mathrm{K}}\right)$ contains the $K$ largest eigenvalues of $\boldsymbol{R}$ in descending order, and $\boldsymbol{U}_{S}$ contains the corresponding orthonormal eigenvectors. $\boldsymbol{\Lambda}_{n}=\sigma^{2} \boldsymbol{I}_{N-K}$ contains another $N-K$ eigenvalues of $\boldsymbol{R}$ and $\boldsymbol{U}_{n}$ contains the corresponding $N-K$ orthonormal eigenvectors. The column vectors of $\boldsymbol{U}_{s}$ and $\boldsymbol{U}_{n}$ span two orthogonal subspaces, namely, the signal subspace and the noise subspace with $\boldsymbol{U}_{s}^{T} \boldsymbol{U}_{n}=0$.
In [17], Zhou et al. have proposed a new blind adaptive multiuser detection scheme based on a hybrid of Kalman filter and subspace estimation. The detector can be expressed as an anchored signal in the signal subspace as follows

$$
c_{1}=s_{1}+s_{1 \text { null }} \boldsymbol{w}_{1}
$$

Subject to

$$
\boldsymbol{c}_{1}^{T} \boldsymbol{s}_{1}=1
$$

Where the column vectors of the matrix $\left[\boldsymbol{s}_{1} \boldsymbol{s}_{1 \text { null }}\right]$ compose

\begin{tabular}{|c|}
\hline $\begin{array}{l}\text { Step 1: } \\
\text { Signal Subspace Estimation: }\end{array}$ \\
\hline $\begin{array}{l}\text { *Compute autocorrelation matrix } \boldsymbol{R} \text { for a batch of } \mathbf{J} \\
\text { symbols } \\
\qquad \boldsymbol{R}=\frac{1}{J} \sum_{j=1}^{J} \boldsymbol{r}_{j} \boldsymbol{r}_{j}^{T}\end{array}$ \\
\hline $\begin{array}{l}\text { * Perform eigenvalue decomposition of } \boldsymbol{R} \\
\qquad \boldsymbol{R}=\boldsymbol{U} \boldsymbol{\Lambda} \boldsymbol{U}^{T}=\boldsymbol{U}_{s} \boldsymbol{\Lambda}_{s} \boldsymbol{U}_{s}^{T}+\boldsymbol{U}_{n} \boldsymbol{\Lambda}_{n} \boldsymbol{U}_{n}^{T}\end{array}$ \\
\hline $\begin{array}{l}\text { * From matrix } \\
\qquad \boldsymbol{Z}=\left[\begin{array}{llll}\boldsymbol{s}_{1} & \boldsymbol{u}_{1} & \ldots & \boldsymbol{u}_{K-1}\end{array}\right] \\
\text { where } \\
\boldsymbol{u}_{i} \text { is the } i^{\text {th }} \text { column vector of } \boldsymbol{U}_{S}, \forall i=1, \ldots, K-1\end{array}$ \\
\hline $\begin{array}{l}\text { * Apply Gram-Schmidt method on } \boldsymbol{Z} \text { to obtain an } \\
\text { orthonormal matrix } \\
\qquad \boldsymbol{Y}=\left[\begin{array}{llll}\boldsymbol{s}_{1} & \boldsymbol{y}_{1} & \ldots & \boldsymbol{y}_{K-1}\end{array}\right]\end{array}$ \\
\hline $\begin{array}{l}\text { * Let } \\
\qquad \boldsymbol{s}_{1 \text { null }}=\left[\begin{array}{lll}\boldsymbol{y}_{1} & \ldots & \boldsymbol{y}_{K-1}\end{array}\right] \\
\text { be the null signal subspace of } \boldsymbol{s}_{1}\end{array}$ \\
\hline $\begin{array}{l}\text { Step 2: } \\
\text { Kalman Filtering Estimation in Symbol-rate Adaptation: }\end{array}$ \\
\hline$*$ For $n=1,2, \ldots$ \\
\hline
\end{tabular}
the signal subspace basis set and $\boldsymbol{w}_{1}$ is a weight vector. Now, $\boldsymbol{s}_{1 \text { null }}$ its columns span the null space of $\boldsymbol{s}_{1}$, i.e. $\boldsymbol{s}_{1}^{T} \boldsymbol{s}_{1 \text { null }}=0$, and they are orthonormal, i.e. $\boldsymbol{s}_{1 \text { null }}^{T} \boldsymbol{s}_{1 \text { null }}=$ $\boldsymbol{I}_{K-1}$. Since $\boldsymbol{s}_{1}$ is assumed to be known and $\boldsymbol{s}_{1 \text { null }}$ can be obtained, for example, by applying eigenvalue decomposition (EVD) to the autocorrelation matrix $\boldsymbol{R}$.

In order to find the optimal weight vector $\boldsymbol{w}_{1}$ that minimizes the MAI. It is demonstrated this vector can be estimated by using the Kalman filter method [2]. The Subspace Kalman blind adaptive algorithm is summarized in Table-1.

Table -1: Subspace-Based Kalman Filtering Estimation for Blind Multiuser Detection 


* Implement Kalman filter algorithm according to
(25)-(27)
* Signal detection
\[ \boldsymbol{c}_{1}(n)=\boldsymbol{s}_{1}+\boldsymbol{s}_{1 \text { null }} \widehat{\boldsymbol{w}}_{\text {opt } 1}(n) \]
* End
$\hat{b}_{1}(n)=\operatorname{sgn}\left(\boldsymbol{c}_{1}^{T}(n), \boldsymbol{r}(n)\right)$

\section{COMPUTATION COMPLEXITY}

We now compare the computational complexity of LMS [3], RLS [6], Kalman [2], and Subspace Kalman blind adaptive algorithms [17]. The computational complexities of four algorithms are compared in term of the number of multiplications and additions per adaptation iteration as shown in Table-2.

Table -1: Comparison of Computational Complexity

\begin{tabular}{|l|c|}
\hline \multicolumn{1}{|c|}{ Algorithm } & $\begin{array}{c}\text { Computational } \\
\text { Complexity }\end{array}$ \\
\hline LMS algorithm [3] & $\mathrm{O}(N)$ \\
\hline RLS algorithm [6] & $\mathrm{O}\left(N^{2}\right)$ \\
\hline Kalman filtering algorithm [2] & $\mathrm{O}\left(N^{2}\right)$ \\
\hline $\begin{array}{l}\text { Subspace Kalman filtering } \\
\text { algorithm [17] }\end{array}$ & $\mathrm{O}(N K)$ \\
\hline
\end{tabular}

Generally, $N \gg>K$; thus, Subspace Kalman has much less computational complexity than the RLS and Kalman filtering algorithms.

\section{SIMULATION RESULTS}

In this section, several simulation results that compare among the four algorithms for blind multiuser detection are presented.

As a figure of merit for assessing the MAI suppression capability of the blind LMS, RLS, Kalman filtering, and subspace-based Kalman filtering algorithms, the timeaveraged SIR (in decibels) at the $n^{\text {th }}$ iteration is given by [3]

$$
\operatorname{SIR}(n)=10 \log \frac{\sum_{l=1}^{M}\left(\boldsymbol{c}_{1 l}^{T}(n) \boldsymbol{s}_{1}\right)^{2}}{\sum_{l=1}^{M} \boldsymbol{c}_{1 l}^{T}(n)\left(\boldsymbol{r}_{l}(n)-b_{1, l}(n) \boldsymbol{s}_{1}\right)^{2}}
$$

Where $M$ is the number of independent runs, and the subscript $l$ indicates that the associated variable depends on the particular run. All signal energies are given in decibels relative to the background noise variance $\sigma^{2}$, i.e., the SNR of user $k$ is defined by $S N R=10 \log \left(E_{k} / \sigma^{2}\right)$, where $E_{k}=A_{k}^{2}$ is the bit energy of user $k$. In all simulations, user 1 is assumed to be the desired user that has the unit energy $A_{1}^{2}=1$ and an $S N R$ of $20 \mathrm{~dB}$ (i.e., $\sigma^{2}=0.01$ ), and the processing gain $N=31$. In the following, the data in each plot are the average over 500 independent runs.

\subsection{Convergence Rate Comparison}

In Example 1, DS-CDMA systems in a Gaussian channel are simulated, and there are nine multiple-access interfering users among which five users have an SNR of $30 \mathrm{~dB}$ each, three users have SNR of $40 \mathrm{~dB}$ each, and another user has an SNR of $50 \mathrm{~dB}$, i.e., $A_{2}^{2}=\ldots=A_{6}^{2}=10, A_{7}^{2}=A_{8}^{2}=A_{9}^{2}=100$, and $A_{10}^{2}=1000$. Then, from (9), it follows that the step size should satisfy $\mu<1.47 \times 10^{-3}$, and thus, $\mu=3 \times 10^{-4}$ was used in the LMS algorithm. When applying the RLS algorithm, the initial value $\boldsymbol{R}^{-1}(0)=\delta^{-1}$ Itakes $\delta=0.01$, and the forgetting factor $\lambda=0.997$ is taken. In Kalman filtering, we used, the estimate $\hat{\zeta}_{\text {min }}=1$ in $(25)$,

The time-averaged SIR versus iteration numbers for the four algorithms is plotted in Fig. 1. It is seen that the performance of the subspace-based Kalman filtering algorithms outperform the rest. When $n$ is sufficiently large subspace-based Kalman filtering algorithm approach $\mathrm{SNR}=19 \mathrm{~dB}$. This means that the MAI in the SIR has been eliminated almost completely. However, note that the subspace-based algorithm achieves this near-optimum performance at a significantly reduced computational complexity compared with the full-rank algorithm. Fig. 2 shows the mean square error (MSE) versus iteration number (time) for the four algorithms applied in a synchronous CDMA system. The most slow algorithm is the LMS while the other three algorithms reach the minimum MSE faster than LMS.

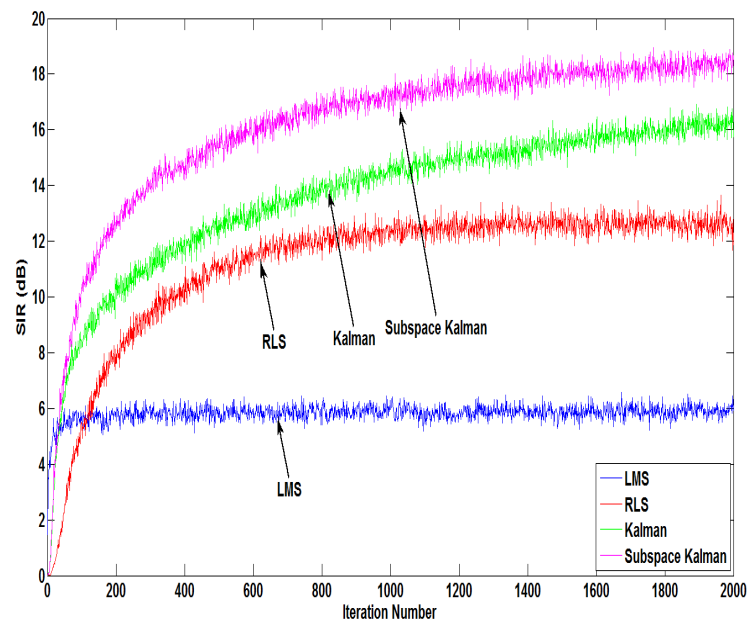

Fig -1: Time-averaged SIR versus time for 500 runs when using the four algorithms to a synchronous CDMA system with processing gain $N=31$. 


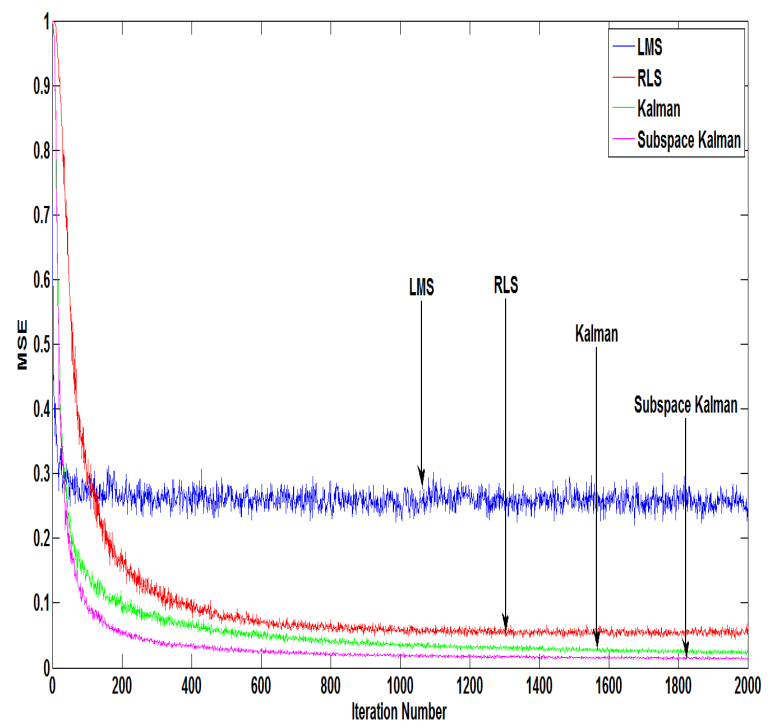

Fig -2: MSE versus time for 500 runs when using the four algorithms to a synchronous CDMA system with processing gain $N=31$.

\subsection{Tracking Dynamical Environment}

In Example 2, we compare the tracking capabilities of the LMS, RLS, Kalman filtering, and subspace-based Kalman filtering algorithms in a dynamical environment with a timevarying number of users for DS-CDMA systems in a Gaussian channel. When $n<600$, the configuration is the same as Example 1. At $n=600$, three interfering users with SNR of $40 \mathrm{~dB}$ are added to the CDMA system at the same time. At $n=1200$, four interfering users with SNR of $40 \mathrm{~dB}$ and one interfering user with SNR of $50 \mathrm{~dB}$ are removed from the system. The subspace-based Kalman filter use projection approximation subspace tracking with deflation (PASTd) algorithm to track the rank and signal subspace with the forgetting factor $\beta=0.997$. Fig. 3 show the tracking behaviors of the four blind adaptive algorithms in a synchronous system.

In Fig. 3, It is also seen that the Kalman filter tracks faster than the subspace approaches due to the reason that in the subspace tracking strategy, the subspace-based algorithm includes two adaptation phases, that is, adaptive subspace tracking and then adaptive signal detection, whereas the fullrank detector includes only one adaptation phase.

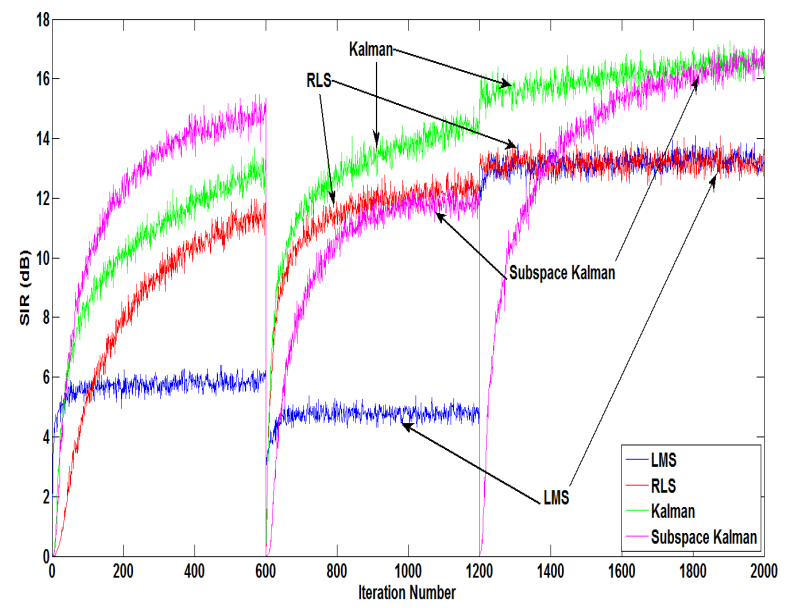

Fig -3: Time-averaged SIR versus time for 500 runs when using the four algorithms to a synchronous CDMA system with processing gain $N=31$ and the time-varying number of users.

\subsection{Slowly Time-Varying Environment}

Example 3 is a synchronous DS-CDMA system in a Rayleigh fading channel. We assume a single-path Rayleigh-fading channel with a Doppler frequency of $22 \mathrm{~Hz}$, which is obtained based on Jakesmodel [18]. The signal model is expressed by

$$
\begin{array}{r}
r(n)=\sum_{k=1}^{K} A_{k} b_{k}(n) h_{k} s_{k}(n)+\sigma v(n), \\
n=0,1, \ldots, T_{s}-1
\end{array}
$$

Where $h_{k}$ is the channel coefficient for the $k^{\text {th }}$ user and is a random variable following Rayleigh distribution The convergence curves for the subspace-based Kalman filter, the Kalman filter and the RLS are plotted in Fig. 4. The results show that all algorithms can track the slow channel fluctuation, and the subspace-based Kalman filtering detector still yields better performance than the other two detectors. The MSE curves of the three algorithms are shown in Fig. 5. The curves show that all of them have the same residual error while the subspace-based Kalman filter is the faster algorithm.

\subsection{BER Performance Comparison}

In Example 4, we evaluate the BER performance of the four algorithms versus SNR in Rayleigh fading channel, the configuration is the same as Example 1. The receivers process 10000 symbols and averaged over 100 independent runs for all BER simulations. The results in Fig. 6 indicate that the subspace-based Kalman filter and the full-rank Kalman filtering algorithms outperform the RLS and LMS algorithms. The performance of the subspace-based Kalman filter 
algorithm is close to the Kalman filtering algorithm but with much lower complexity.

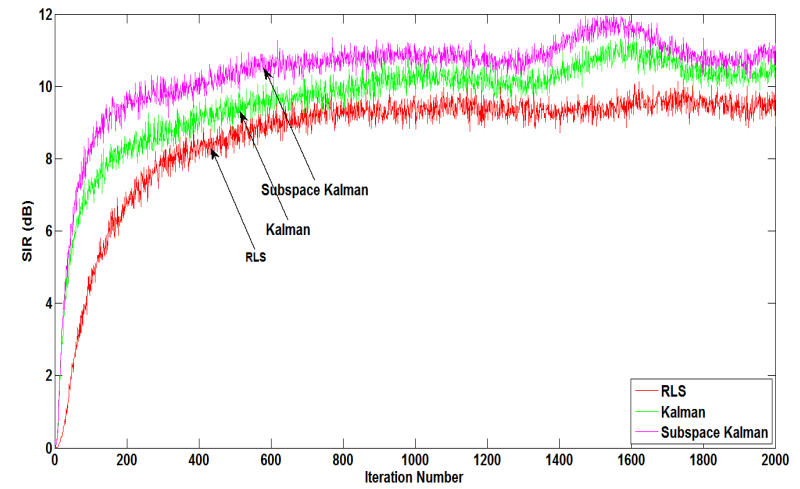

Fig -4: Time-averaged SIR versus time for 500 runs when using the three algorithms with $\lambda=0.997$ to a synchronous CDMA system in Rayleigh fading channel; the processing gain is $N=31$.

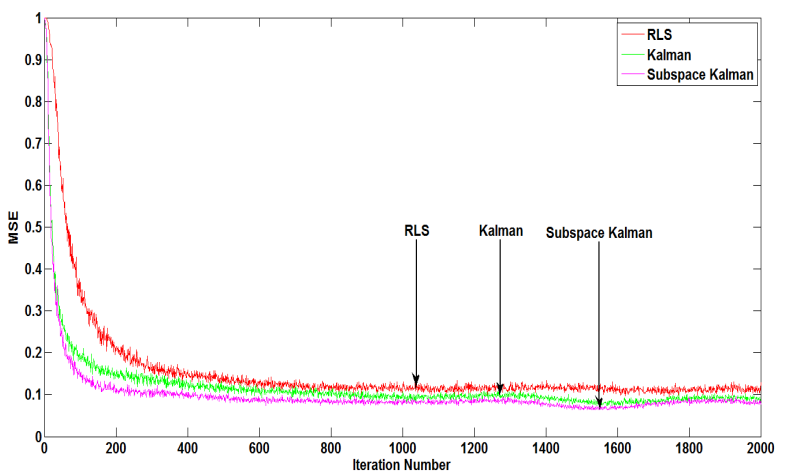

Fig -5: MSE versus time for 500 runs when using the three algorithms with $\lambda=0.997$ to a synchronous CDMA system in Rayleigh fading channel; the processing gain is $N=31$.

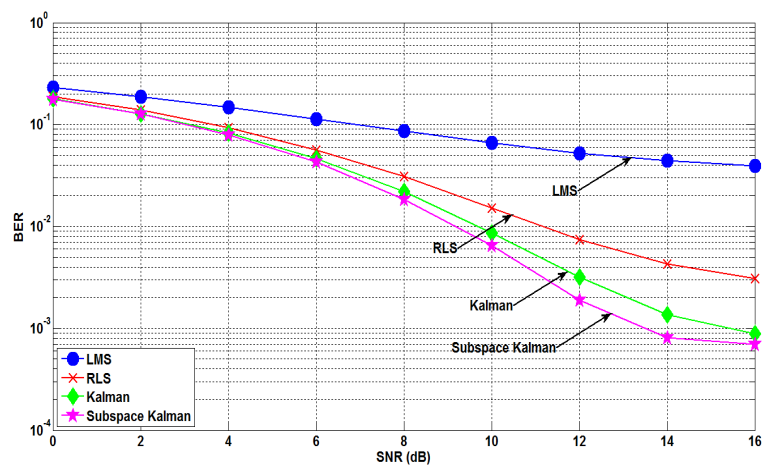

Fig -6: BER versus SNR for 100 runs when using the four algorithms to a synchronous CDMA system in Rayleigh fading channel.

\section{CONCLUSIONS}

In this paper, we have analyzed the SIR and MSE performance of four blind-adaptive algorithms. Subspace-based Kalman filter has near-far resistant, lower computational complexity, and better convergence performance compared with another algorithms. It is effective in both AWGN channel and slowly time-varying Rayleigh-fading channel. It is also a blind detection method in a stricter sense because it is less conditioned on the knowledge of the signal amplitude of the desired user. Adaptation in the dynamic environment with variable number of users is enabled by seamlessly integrating a subspace tracking methodology at the cost of slight increment in computational complexity.

\section{REFERENCES}

[1]. S. Kapoor, S. Gollamudi, S. Nagaraj, and Y. -F. Huang, "Adaptive multiuser detection and beamforming for interference suppression in CDMA mobile radio systems," IEEE Trans Veh. Technol., vol. 48, pp. 1341-1355, Sept. 1999.

[2]. X. D. Zhang and W. Wei, "Blind adaptive multiuser detection based on Kalman filtering," IEEE Trans. Signal Process., vol. 50, no. 1, pp. 87-95, Jan. 2002.

[3]. M. L. Honig, U. Madhow, and S. Verdu, "Blind adaptive multiuser detection," IEEE Trans. Inform. Theory, vol. 41, pp. 944-960, July 1995.

[4]. S. Roy, "Subspace blind adaptive multiuser detection for CDMA," IEEE Trans. Commun., vol. 48, pp. 169-175, Jan. 2000.

[5]. D. S. Chen and S. Roy, "An adaptive multiuser receiver for CDMA systems," IEEE J. Select. Areas Commun., vol. 12, pp. 808-816, June 1994.

[6]. H. V. Poor and X. Wang, "Code-aided interference suppression for DS/CDMA communications, Part II: Parallel blind adaptive implementations," IEEE Trans. Commun., vol. 45, pp. 1112-1122, Sept. 1997.

[7]. S. Haykin, Adaptive Filter Theory, 3rd ed. Englewood Cliffs, NJ: Prentice- Hall, 1996.

[8]. A. H. Sayed and T. Kailath, "A state-space approach to adaptive RLS filtering," IEEE Signal Processing Mag., vol. 11, pp. 18-60, July 1994.

[9]. B. D. O. Anderson and J. B. Moore, Linear Optimal Control. Englewood Cliffs, NJ: Prentice-Hall, 1979.

[10]. G. C. Goodwin and K. S. Sin, Adaptive Filtering, Prediction and Control. Englewood Cliffs, NJ: Prentice-Hall, 1984.

[11]. R. Iltis and L. Mailander, "An adaptive multiuser detector with joint amplitude and delay estimation," IEEE J. Select. Areas Commun., vol. 12, pp. 774-785, June 1994.

[12]. S. H. Isabelle and G. W. Wornell, "Efficient multiuser detectors for intersymbol interference channels," in Proc. SPAWC, 1997, pp. 277-280.

[13]. T. J. Lim, L. K. Rasmussen, and H. Sugimoto, "An asynchronous multiuser CDMA detector based on the Kalman 
filter," IEEE J. Select. Areas Commun., vol. 16, pp. 17111722, Sept. 1998.

[14]. T. J. Lim and Y. Ma, "The Kalman filter as the optimum linear minimum mean squared error multiuser CDMA detector," IEEE Trans. Inform. Theory, vol. 46, pp. 25612566, July 2000.

[15]. P.-W. Fu and K. C. Chen, "Linear-complexity equalized multiuser receivers for wideband CDMA in time-varying channels," in Proc. Veh. Technol. Conf., 1999, pp. 23382342.

[16]. X. D. Wang and H. V. Poor, "Blind multiuser detection: A subspace approach," IEEE Trans. Inf. Theory, vol. 44, no. 3, pp. 677-690, Mar. 1998.

[17]. H. Zhou, W. L. Woo, and B. S. Sharif, "Subspace-based blind adaptive multiuser detection using Kalman filter," IEEE Proc. Commun., 152(3):301-310, 2005.

[18]. Jakes, W.C. (ed.): 'Microwave mobile communications' (Wiley-IEEE Press, New York, 1994)

\section{BIOGRAPHIES}

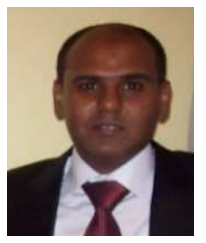

KhalifaHunkireceived the B.S. degree in electronic engineering from Engineering Academy Tajoura, Tajoura, Libya, in 2001 and the M.Sc. degree in electronic engineering from the Military Technical College, Cairo, Egypt, in 2008.Currently, he is working toward the Ph.D. degree with the Department of Electronic Warfare, Military Technical College, Egypt. His research interests include multiuser detection, and adaptive signal processing.

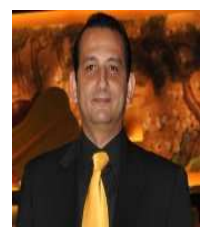

Ehab M. Shaheenwas born in Cairo, Egypt, on March 16, 1973 Obtained the B.Sc. and M.Sc. in electrical engineering in 1995 and 2000 respectively, both from Military Technical College in Cairo, Egypt, and the Ph.D. in Computer and Electrical Engineering from Carleton University, Ottawa, Ontario, Canada in 2010.He joined the Military Technical College in 2011 and he is currently astaff in the Electronic Warfare Department where his current research interests include ultra-wideband (UWB) radio communications, spread-spectrum and secure communication signalsand digital communications over multipath fading channels.

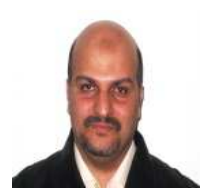

Mohamed Soliman received the BEng. degree in Comunication and the Master of Science degree from the Military Technical College, Egypt 1993 and 2001, respectively. Between 1993 and 2006, he was an engineering officer in Egyptian armed forces. Since 2006, he has been a PhD student in the School of Electrical and Electronic Engineering, University of Manchester. $\mathrm{He}$ received his $\mathrm{PhD}$ from the Manchester University in January 2009.

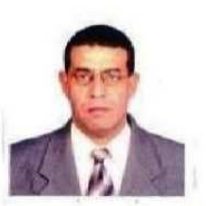

K.A. El-Barbary received the B. S. degree (with Average Grade Very Good) in Electric Engineering from Military Technical College, Egypt , the M.S. degree in Electric Engineering from Military Technical College, Egypt, the Ph.D. degree in Communications from George Washington University, Washington DC, USA, in 1981, 1986 and 1993, respectively. He was faculty member at Military Technical College, Egypt in the Electric Engineering Department (Chair of communications and Electronic warfare) (1994-2010). There he became a professor of Electric Engineering (2009), Head of Electronic Warfare Engineering Department (2000), and Head of Electrical Engineering Department (2009). He has been a professor of signal and systems and Communications at Suez Canal University, Egypt in Electric Engineering Department. Currently he is the Head of Electric Engineering Department, Suez Canal University, Egypt. His research interests include Statistical signal and array processing and related applications in communications, radar, Electronic Intelligence, Multi user Detectors and wideband systems. 